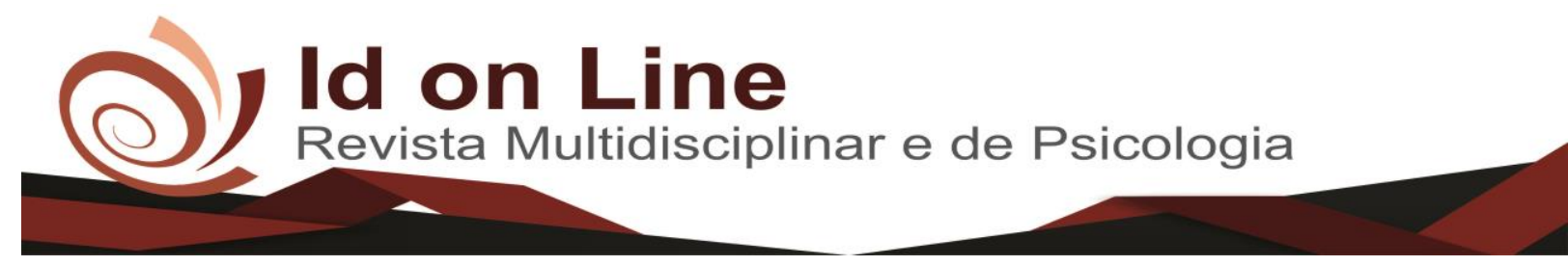

Artigo de Revisão

\title{
As Implicações da Política Nacional de Atenção Básica (PNAB) na Dinâmica dos Serviços de Saúde
}

Juvenildo Santos Ferreira'; Luiz Eduardo de Góes Ladeia²

\begin{abstract}
Resumo: Os avanços obtidos em Saúde Pública, no Brasil, são inquestionáveis e possibilitaram a qualificação e ampliação do acesso à saúde. Apesar das conquistas, medidas adotadas pelo Governo tem levado ao empobrecimento do Sistema Único de Saúde (SUS). Entre estas, destaca-se a Nova Política Nacional da Atenção Básica (PNAB). Diante da relevância desta temática, da ausência de diálogo prévio a reformulação da PNAB com categorias de controle social e das implicações diretas dessa reformulação no modelo de trabalho e assistência à Saúde efetuou-se essa revisão de literatura com o objetivo de compreender as principais implicações da nova PNAB nos Serviços de Saúde. Para elaboração da revisão efetuou-se busca por artigos nas bases de dados. Após leitura e análise dos artigos foram selecionados os artigos publicados entre 2012 e 2018 que se relacionavam ao tema. Salienta-se que a nova PNAB, criada em 2017, apesar de manter a essência textual apresenta inovações em seu conteúdo. A nova PNAB propôs algumas mudanças consideradas ineficazes, podendo levar ao insucesso desta. A literatura demonstra haver possível deficiência na expansão da saúde e com o sistema público, o que pode afetar o atendimento das necessidades da população. Há um consenso entre os autores sobre os impactos negativos a respeito da PNAB, principalmente sob a Estratégia de Saúde da Família, no entanto, observa-se a relevância de recursos estratégicos como a participação social da população na garantia dos direitos adquiridos.
\end{abstract}

Descritores: Atenção Primária à Saúde; Política de Saúde; Política Pública.

\section{The Implications of the Basic Attention National Policy (PNAB) in the Dynamics of Health Services}

\begin{abstract}
The advances obtained in Public Health in Brazil are unquestionable and enabled the qualification and expansion of access to health. Despite the achievements, measures adopted by the Government have led to the impoverishment of the Unified Health System (SUS). Among them, the New National Policy for Primary Care (PNAB) stands out. Given the relevance of this issue, the absence of prior dialogue to reformulate the PNAB with categories of social control and the direct implications of this reformulation in the model of work and health care, a literature review was made with the objective of understanding the main implications of the new PNAB in the Health Services. For the preparation of the review, was conducted a search for articles in the article databases. After reading and analyzing the articles, articles published between years 2012 and 2018 and related to the topic were selected. It should be noted that the new PNAB, created in 2017, despite maintaining the textual essence, presents innovations in its content. The new BANP proposed some changes considered ineffective, which could lead to its failure. The literature shows that there is a possible deficiency in relation to health expansion and to the public system, which can affect the needs of the population. There is a consensus among the authors about the negative impacts on PNAB, especially under the Family Health Strategy, however, the relevance of strategic resources such as the social participation of the population in the guarantee of acquired rights is observed.
\end{abstract}

Keywords: Primary Health Care; Health Policy; Public Policy.

\footnotetext{
${ }^{1}$ Graduando em Odontologia pela Faculdade Independente do Nordeste; autor para correspondência. Endereço: Rua Vasco da Gama, ${ }^{\circ}$ 151, Alto Maron. Vitoria da Conquista - Bahia. Cep: 45005246. Email: juvenildo2000@ yahoo.com.br.

${ }^{2}$ Professor Especialista do curso de Odontologia pela Faculdade Independente do Nordeste
} 


\section{Introdução}

Os avanços obtidos pelos investimentos em Saúde Pública, no Brasil, são inquestionáveis e possibilitaram, ao longo de décadas, a ampliação do acesso à saúde, a universalização da assistência e melhoria na oferta de serviços de saúde. Essa melhoria nas condições de saúde do brasileiro possui relação direta com a criação do Sistema Único de Saúde (SUS), no início na década de 90, a partir do reconhecimento da saúde como direito fundamental e dever do estado através da Constituição Federal de 1988 (VIEIRA e BENEVIDES, 2016).

O SUS se constitui em um conjunto de ações e serviços, em saúde, prestados por Instituições Públicas organizadas nos diversos níveis e é mantido pelo Poder Público. Apresenta como princípios essenciais a universalidade, a igualdade, a integralidade, a descentralização e a participação popular (VIEIRA e BENEVIDES, 2016). Para que os serviços do SUS sejam fornecidos o Ministério da Saúde, através de uma série de portarias, define o modo de atuação do Estado brasileiro através da delimitação políticas públicas, com corresponsabilização de outras esferas do governo, preconizando-se a organização da oferta de serviços de saúde de qualidade (DOMINGOS; NUNES, CARVALHO e MENDONÇA, 2016).

Se por um lado os avanços nos serviços foram exitosos, por outro, a crise vivenciada pelo capitalismo teve suas implicações no SUS (MENDES, 2015). Várias estratégias, recentes, adotadas pelo Governo têm levado ao seu sucateamento (ROSSI e DWECK, 2016) e "desconstrução" do SUS. Destaca-se, neste contexto, a aprovação da Emenda Constitucional 95/2016, que apresenta novo regime fiscal para os próximos 20 anos com limitação dos gastos e investimentos em políticas públicas e a nova Política Nacional da Atenção Básica (PNAB), em 2017 (MARIANO, 2017; ROCHA e MACÁRIO, 2017; MOROSINI e FONSECA, 2017) .

A PNAB estabelece diretrizes para organização da Atenção Primária enfatizando a mesma como coordenadora da Rede de Atenção em Saúde (DOMINGOS; NUNES, CARVALHO e MENDONÇA, 2016). Esta política já foi submetida a várias modificações, de maneira que a mais recente foi aprovada em 2017 e incorpora uma série de inovações (FONTENELLE, 2012; BRASIL, 2017).

No que se refere à nova versão da $\mathrm{PNAB}$, as principais problemáticas tem sido a carteira mínima de serviços ofertados, fusão dos Agentes Comunitários de Saúde e Agentes de Endemias em um único profissional e atribuição de remuneração adicional, dentre outros (MOROSINI e FONSECA, 2017; BRASIL, 2017). Todas estas mudanças implicam diretamente sobre os Serviços de Saúde da Atenção Básica e sobre o modelo de saúde vigente restringindo a prestação de assistência a um modelo de saúde focado na doença não preconizado pela Ministério da Saúde (MOROSINI e FONSECA, 2017). 
Partindo-se do pressuposto que vivenciar o SUS envolve experienciar e debater, de forma organizada, articulada (MARANHÃO e MATOS, 2017) e que não houve tempo hábil para discussão entre as categorias de controle social no que tange a reformulação da PNAB e diante da relevância desta temática e das implicações diretas da reformulação da PNAB sobre as relações de trabalho e nos serviços de Saúde foi proposta esta revisão de literatura com o objetivo de compreender as principais implicações da nova PNAB na dinâmica dos Serviços de Saúde.

A revisão de literatura apresenta-se como um recurso fundamental na perspectiva que oferece uma análise crítica diante da Nova Política Nacional da Atenção Básica. A opção por revisar os conhecimentos sobre as implicações da PNAB na dinâmica dos Serviços de Saúde representa um papel diversificado na medida em que os trabalhos científicos publicados sobre o assunto são escassos. Portanto, o objetivo do presente trabalho é compreender as principais implicações da reformulação da PNAB na dinâmica dos Serviços de Saúde por meio de uma revisão de literatura.

\section{Métodos}

Efetuou-se uma revisão de literatura a respeito da Política Nacional da Atenção Básica nas bases de dados MEDLINE, LILACS e GOOGLE SCHOLAR. Foram estabelecidos como critérios de inclusão: artigos com resumo e texto completo disponíveis em português ou inglês, publicados entre 2012 e 2018, abordando o PNAD, em qualquer localidade. Os critérios de exclusão consistiram em artigos que não se relacionavam ou não debatiam o tema. Após leitura e análise dos artigos e seleção dos artigos pertinentes, efetuou-se a confecção da presente revisão de literatura. Ao final da revisão, compuseram o presente trabalho 21 artigos e portarias.

\section{Revisão de Literatura}

São inquestionáveis os avanços obtidos, na Saúde Pública, ao longo de décadas e que permitiram a ampliação do acesso à saúde através da universalização da assistência com maior oferta de serviços em saúde (DOMINGOS; NUNES, CARVALHO e MENDONÇA, 2016). 
De maneira sucinta pode-se afirmar que o progresso nas condições de saúde da população brasileira, nas últimas décadas, foi resultado de ações sobre determinantes em saúde, devido à Reforma que culminou na Criação do Sistema Único de Saúde (SUS) e da Constituição Federal de 1988, (VIEIRA e BENEVIDES, 2016).

A partir da Constituição de 1988 o direito à saúde passou a ser reconhecido como direito de todos e dever do Estado sendo implementado um conjunto de ações para que esta premissa fosse efetivada. Neste sentido, diversos programas e estratégias foram adotados pelo Ministério da Saúde para fortalecimento da Atenção Básica (DOMINGOS; NUNES, CARVALHO e MENDONÇA, 2016).

Neste contexto surge a Política Nacional da Atenção Básica (PNAB) que constitui um conjunto de diretrizes para reorganização da Atenção Primária e define a Atenção Básica como organizadora das Redes de Atenção à Saúde sendo aprovada pela Portaria 2.488/2011, em 30 de agosto de 2017 (DOMINGOS; NUNES, CARVALHO e MENDONÇA, 2016; MOROSINI, FONSECA e LIMA, 2018). A PNAB é responsável pela coordenação das ações integrais que envolvem estratégias horizontais como ações programáticas, vigilância, assistenciais, multidisciplinaridade e interdisciplinaridade. Também envolve estratégias verticais nos diferentes níveis da Rede de Atenção à Saúde, sendo a Estratégia de Saúde da Família prioridade na reorganização dessa rede (ALMEIDA e SANTOS, 2016; MOREIRA e O’DWYER, 2013).

A PNAB amplia a cobertura de serviços, programas e territórios visando atender as necessidades populacionais. Esta Política é pautada em princípios norteadores do SUS como universalidade, acessibilidade, definição de atribuições/funções, continuidade do cuidado, humanidade, responsabilização, integralidade, equidade e participação social (BARBIANI, DALLA NORA e SHAEFER, 2016).

A PNAB desenvolveu-se com expressividade ao permitir conciliar questões discursivas sobre Atenção Primária/Atenção Básica. Entre a política oficial e realidade local dos serviços surge a PNAB com o intuito de operacionalizar os serviços em suas diversificadas redes ${ }^{15}$. Essa política foi submetida a modificações, sendo recentemente aprovada uma nova versão que incorpora inovações significativas (MOROSINI e FONSECA, 2017; FONTENELLE, 2012; CECILIO e REIS, 2018). 
A PNAB de 2006 a 2013, de maneira geral, buscou solucionar problemas vigentes na Atenção Primária à Saúde. A PNAB de 2011 fortaleceu a centralização da Atenção Primária, a organização em rede, ampliou as ações do NASF, reconheceu as especificidades da equipe e necessidades diferenciadas dos moradores de rua e populações ribeirinhas e trouxe uma nova perspectiva de ação intersetorial através do Programa Saúde na Escola (PSE). Agregou-se à Atenção Básica o Programa Nacional de Melhoria do Acesso e da Qualidade da Atenção Básica (PMAQ-AB) e o Programa Mais Médicos. Sob uma perspectiva ampliada observou-se uma convergência entre as ações propostas com os princípios do Sistema Único de Saúde (ALMEIDA, 2018).

Contraditoriamente às versões anteriores, a nova PNAB criada em 2017, apesar de manter a essência textual apresenta inovações em seu conteúdo (FONTENELLE, 2012). A nova PNAB propôs mudanças descompassadas podendo levar ao insucesso dessa política devido à possibilidade de interrupção da continuidade assistencial e vínculo - atributos primordiais no modelo de Atenção a Saúde (ALMEIDA, 2018). Essa PNAB evidencia o descompromisso com a expansão da saúde e com o sistema público, comprometendo o atendimento das necessidades da população (MOROSINI, FONSECA e LIMA, 2018; ALMEIDA, 2018) .

Essa nova versão da PNAB discute aspectos como carteira mínima de serviços ofertados, fusão entre Agentes Comunitários de Saúde e Agentes de Endemias (categoria única profissional), atribuição de remuneração adicional em atividades de monitoramento, avaliações gerando "caráter punitivo", flexibilização da carga horária do médico na Estratégia de saúde da Família, incorporação dos Núcleos de Apoio à Saúde da Família (NASF) às equipes ribeirinhas e Unidades fluviais (NASF-AB) bem como a presença do profissional de saúde bucal das Equipes de Saúde da Família nas Equipes de Agentes Comunitários de Saúde (ACS), dentre outras mudanças (MOROSINI e FONSECA, 2017; FONTENELLE, 2012; BRASIL, 2017).

Nos aspectos conceituais a nova PNAB inova ao reconhecer que a Atenção Primária em Saúde se articula com outros serviços através de uma rede de atenção à saúde (FONTENELLE, 2012). A oferta de uma carteira mínima de serviços que atenda as necessidades e especificidades locais também tem sido mencionada como algo preocupante visto que oferece serviços de acordo com as demandas locais, reforçando as desigualdades, intensificando o vínculo precário e estratificando e simplificando os serviços de saúde ( MOROSINI e FONSECA, 2017; ALMEIDA, 2018). 
Outra mudança proposta foi à fusão do ACS ao ACE (Agente de Combate em Endemias) na figura do Agente de Saúde. Segundo a PNAB as ações de vigilância e da Atenção Básica necessitam ser associadas para maior sucesso das ações no território. Essa mudança promove uma descaracterização da função do ACS assumindo maior atuação na prevenção de doenças (MOROSINI e FONSECA, 2017; MOROSINI, FONSECA e LIMA, 2018). A expectativa é de corte de custos e intensificação do trabalho. Ao vislumbrar esta união desconsidera-se que a integração entre diversas áreas requer modificações significativas no processo de trabalho (MOROSINI e FONSECA, 2017).

Em relação à relativização da cobertura, a nova PNAB discorre sobre o fim da obrigatoriedade da cobertura universal dos ACS, sendo exigida cobertura de $100 \%$ apenas em populações vulneráveis e em áreas de risco, sendo estas definidas pelo gestor em conjunto com a Equipe da Atenção Básica e Conselho Municipal ou Local (MOROSINI, FONSECA e LIMA, 2018; CECILIO e REIS, 2018) . O número de ACS por Equipe, na nova versão da PNAB, não é definido de acordo com dados demográficos e legislação vigente, fato que gera inconformação a respeito do papel do ACS e universalidade de acesso (MOROSINI, FONSECA e LIMA, 2018).

Os padrões essenciais são ações e procedimentos básicos que deveriam estar garantidos em todo país. Já os padrões ampliados correspondem a procedimentos e ações capazes de elevar a qualidade e o acesso, conforme necessidades locais e gestão. A PNAB recomenda os padrões ampliados, no entanto, apenas os padrões essenciais serão realizados com compromisso. Desta maneira a nova PNAB evidencia característica seletiva e fragmentada da Atenção Primária da Saúde ao ofertar "cuidados mínimos". Ao diferenciar padrões da AB em ações e cuidados a nova PNAB descompromete-se (MOROSINI, FONSECA e LIMA, 2018).

Outro aspecto mencionado na PNAB é a oferta de planos populares e de adesão voluntária. A restrição do acesso bem como a baixa qualidade dos serviços, provavelmente, serão indutores da evasão da classe popular e adesão da classe média a estes planos. Essas ações articuladas encaminham-se para exclusão do SUS e geração de clientela para os planos privados, que por sua vez se mostram incertos quanto à capacidade de suprir as demandas populacionais (MOROSINI, FONSECA e LIMA, 2018).

Assim, a redução da prioridade da ESF, a flexibilização do modelo de atenção e uso de recursos por meio do PAB variável, fragilizam a regulação e comprometem a descentralização 
do SUS. Ao facultar a Gestão a composição das Equipes da Atenção Básica, conforme especificidades locais, desestrutura-se o modelo focado na Atenção Básica e tende a agravar a condição de subfinanciamento do SUS (MOROSINI, FONSECA e LIMA, 2018; PINTO e GIOVANELLA, 2018).

A nova PNAB, também, reconhece que a Equipe mínima deve ser formada por médico, enfermeiro, auxiliar ou técnico de enfermagem, podendo ou não agregar outros profissionais. Nessa PNAB descreve-se que as equipes devem ser compostas pela Gestão municipal conforme necessidades locais, levando em conta o perfil demográfico e epidemiológico que é de sua responsabilidade e a distribuição da carga horária dos profissionais, de forma flexível, mantendo-se a carga horária mínima de 10 horas, podendo haver no máximo três profissionais em mesma categoria (MOROSINI, FONSECA e LIMA, 2018; CECILIO e REIS, 2018; COSTA, 2016). A criação de equipes com dedicação parcial à Atenção Primária e a readequação da equipe profissional com menor carga horária no Serviço tem sido amplamente discutida e criticada devido à possibilidade de fragmentação do processo de trabalho com múltiplos vínculos profissionais e menor vínculo entre a população e a equipe de referência (FONTENELLE, 2012; CECILIO e REIS, 2018; ALMEIDA, 2018). Porém, este recurso apresenta-se como uma alternativa às regiões com maior escassez de profissionais (FONTENELLE, 2012).

Ao eliminar o Agente Comunitário de Saúde da Equipe mínima a Política desonera, financeiramente, a Gestão Municipal e evidencia a preocupação com a redução de despesas como piso salarial e direitos adquiridos pelo vínculo empregatício. No entanto, ao excluir o ACS da Equipe mínima coloca-se em risco as ações de promoção e prevenção de saúde, e sobre os determinantes em saúde essencial para prestação de uma assistência integrada à saúde (MOROSINI, FONSECA e LIMA, 2017; PINTO e GIOVANELLA, 2018).

A flexibilização da carga horária e da cobertura evidenciam pouca preocupação com os efeitos das mudanças no modelo de saúde e grande preocupação com mera redução de custos desfavorecendo-se a longevidade da assistência (MOROSINI, FONSECA e LIMA, 2017; PINTO e GIOVANELLA, 2018).

O texto da nova PNAB descreve a necessidade de inclusão de um Gerente em Atenção Básica, profissional com nível superior não vinculado à Unidade, capaz de promover o 
aprimoramento e qualificação profissional fortalecendo a atenção a saúde da população (BRASIL, 2017).

No que tange ao número de pessoas por ESF a nova PNAB modificou a recomendação de quatro mil para uma população adscrita de dois mil e quinhentos a três mil pessoas para cada Estratégia de Saúde da Família, garantindo-se os princípios e diretrizes da Atenção Básica 9 . Neste sentido, torna-se primordial o estabelecimento de mecanismos para controle desse número máximo de pessoas (FONTENELLE, 2012).

Quanto ao Programa Nacional de Melhoria do Acesso e Qualidade da Atenção Básica (PMAQ-AB) a inovação se deu na avaliação dos indicadores de desempenho que serão avaliados pelo Ministério da Saúde (MS) e os demais por Equipes oriundas de Instituições de Ensino e Pesquisa contratadas pelo MS, sendo submetidos a esta avaliação equipes da atenção básica (FONTENELLE, 2012). Por outro lado, aponta-se um “caráter punitivo" implícito no monitoramento ${ }^{7}$. Embora o processo avaliativo seja essencial para gerar informações e reorientar políticas, a atribuição de remuneração adicional interfere na reflexão, troca de saberes e condições legítimas do processo de trabalho (MOROSINI e FONSECA, 2017).

Se os avanços nos Serviços de Saúde foram exitosos, por outro lado, estratégias recentes adotadas pelo Governo têm gerado críticas a respeito da desconstrução do Sistema Único de Saúde e possibilidade de sucateamento (MENDES, 2015; ROSSI e DWECK, 2016). Além da aprovação da nova PNAB, em 2017, a aprovação da Emenda Constitucional 95/ 2016 com a limitação dos gastos e políticas públicas por vinte anos gerou incertezas sobre a manutenção do sistema vigente e evidencia a crise do capital, além de afetar o SUS com efeitos diretos sobre recursos públicos e apropriação (VIEIRA e BENEVIDES, 2016; MENDES, 2015; ROSSI e DWECK, 2016; MARIANO, 2017; ROCHA e MACÁRIO, 2017; MOROSINI e FONSECA, 2017).

Os desafios enfrentados minimizam o reconhecimento do êxito do SUS e corroboram com a sua desconstrução do SUS (MOROSINI, FONSECA e LIMA, 2017). Em tempos de ameaça cabe a defesa, contundente, ao SUS e a Atenção Primária como pilar essencial na sustentabilidade e prestação de assistência resolutiva, eficaz, equitativa e acessível (ALMEIDA, 2018).

Apesar das limitações organizacionais para prestação de assistência em rede (falhas no sistema de contrareferência e comunicação interprofissional), a Atenção Primária ainda 
estabelece o primeiro contato do paciente e é a porta preferencial do usuário ao sistema (ALMEIDA e SANTOS, 2016; CARVALHO, GIL, COSTA, SAKAI e LEITE, 2018; FERTONANI, PIRES, BIFF e SHERER, 2015). Não obstante, os temores gerados pela nova PNAB são inconsistentes, minimizando o valor atribuído à Saúde da Família no aspecto de organização da Atenção Primária do país (FONTENELLE, 2012).

A PNAB afirma que a Saúde da Família é a prioridade na estruturação dos serviços e organização dos sistemas municipais de saúde. Contudo, diversas fragilidades potenciais na PNAB corroboram com uma prática assistencial centralizada no médico e no tratamento e reabilitação de doenças (FERTONANI, PIRES, BIFF e SCHERER, 2015). Pode-se afirmar que essas mudanças geradas pela nova PNAB e outras ações, em conjunto, impactarão diretamente sobre os Serviços de Saúde da Atenção Básica e modelo de assistência à Saúde vigente (MOROSINI e FONSECA, 2017).

A nova PNAB já é vista como sério risco às conquistas de fortalecimento obtidas pela Atenção Primária, no Brasil, bem como reforça a subtração de direitos (MOROSINI, FONSECA e LIMA, 2017) ${ }^{11}$. Essa nova PNAB tem suscitado críticas de diversas organizações vinculadas ao SUS como a Associação Brasileira de Saúde Coletiva (Abrasco), Centro Brasileiro de Estudos de Saúde (Cebes) e Escola Nacional de Saúde Pública, devido aos retrocessos desta política na Atenção Básica, Atenção Primária à Saúde e Equipe de Saúde da Família (MOROSINI, FONSECA e LIMA, 2017).

Vale destacar que não se tem ainda estudos ou projeções capazes de delimitar o impacto das medidas implementadas pela nova PNAB (CECILIO e REIS, 2018). No entanto, evidenciase a necessidade da participação social para que o debate de aspectos primordiais, como a assistência à saúde e compromisso com a saúde da população, sejam mantidos (MOROSINI e FONSECA, 2017).

Aos usuários e familiares cabe garantir que suas necessidades sejam atendidas, nos diversos pontos de Atenção à Saúde, de forma continuada. Aos profissionais competem propor ações que integrem família, paciente e equipe, atendendo suas peculiaridades de forma articulada e continuada (CECILIO e REIS, 2018; ALMEIDA, 2018). Portanto, permanece o desafio cívico de organizar um sistema de saúde, efetivamente, público e universal (COSTA, 2016). 


\section{Discussão}

A Saúde Pública avançou, significativamente, ao longo das décadas garantindo acessibilidade e universalização dos serviços de saúde o que gerou melhorias nas condições de saúde da população brasileira (VIEIRA e BENEVIDES, 2016; DOMINGOS, NUNES, CARVALHO e MENDONÇA, 2016).

Se por um lado a Constituição Federal de 1988 garantiu o direito à saúde e se na criação do SUS houve um fortalecimento da Atenção Básica e dos serviços de saúde, por outro, estratégias recentes adotadas pelo Governo tem gerado inúmeras críticas a respeito da desconstrução do Sistema Único de Saúde e possibilidade de sucateamento (VIEIRA e BENEVIDES, 2016; ROSSI e DWECK, 2016).

A aprovação da Emenda Constitucional 95/ 2016 com a limitação dos gastos e políticas públicas por vinte anos gerou incertezas sobre a manutenção do sistema vigente ${ }^{1,4-6}$. Associada a essa emenda foi recentemente aprovada nova PNAB, através da Portaria 2.488/2011 em 30 de agosto de 2017 (MOROSINI e FONSECA, 2017; FONTENELLE, 2012; MOROSINI, FONSECA e LIMA, 2017; CECILIO e REIS, 2018).

Apesar das afirmações de que a Atenção Básica é a porta de entrada do serviço de saúde, as incertezas geradas pela nova PNAB são inúmeras ${ }^{8,20,21}$. Dicotomicamente essa PNAB refere que a Saúde da Família é a prioridade na estruturação dos serviços e por outro lado estimula uma prática assistencial hospitalocêntrica, impactando diretamente sobre os Serviços de Saúde e modelo de assistência à Saúde vigente (MOROSINI e FONSECA, 2017; FERTONANI, PIRES BIFF e SCHERER, 2015).

Desta forma, a nova PNAB, submetida a modificações é criticada por diversas organizações vinculadas ao SUS que avaliam a mesma como usurpadora de direitos e promotora de retrocessos dos direitos adquiridos (MOROSINI e FONSECA, 2017; MOROSINI, FONSECA e LIMA, 2017) ${ }^{7,11}$. De maneira geral a PNAB constitui um conjunto de diretrizes responsáveis por organizar a Atenção Básica e Redes de Atenção à Saúde, através de estratégias verticais e horizontais, focadas na Estratégia de Saúde da Família, pautadas nos princípios do SUS (DOMINGOS, NUNES, CARVALHO e MENDONÇA, 2016; ALMEIDA e SANTOS, 2016; MOREIRA e O’DWYER, 2013; CECILIO e REIS, 2018). 
As versões anteriores da PNAB tinham como propósito solucionar problemas vigentes na Atenção Primária à Saúde (ALMEIDA, 2018). Contraditoriamente, a nova PNAB evidencia o descompromisso com a expansão da saúde, com o sistema público e com as necessidades da população (FONTENELLE, 2012; MOROSINI, FONSECA e LIMA, 2017; ALMEIDA, 2018).

Um dos pontos discutidos na nova PNAB é a oferta mínima de serviços, que é o fornecimento de serviços que atendam as necessidades e especificidade. Este quesito, no entanto, tende a reforçar desigualdades e intensificar a estratificação dos serviços de saúde (MOROSINI e FONSECA, 2017; ALMEIDA, 2018).

A diferenciação entre ações padrões essenciais e padrões ampliados evidenciam uma perspectiva seletiva e fragmentada da Atenção Primária focada em "cuidados mínimos" (MOROSINI, FONSECA e LIMA, 2017). A nova PNAB menciona também a oferta de planos populares de adesão voluntária. No entanto, a restrição ao acesso e a baixa qualidade dos serviços induziriam adesão da classe média a estes planos. Vislumbra-se, portanto, que esse conjunto de ações estão, provavelmente, encaminhando para exclusão do SUS (MOROSINI, FONSECA e LIMA, 2017).

A Fusão do ACS e ACE em Agentes de Saúde também é discutida. Com discurso de unificar competências para maior abrangência das ações de vigilância na Atenção Básica, essa ação promove descaracterização da função do ACS (MOROSINI e FONSECA, 2017; MOROSINI, FONSECA e LIMA, 2017). Um dos motivos para esta medida é o corte de custos (MOROSINI e FONSECA, 2017). No entanto, ao eliminar o Agente Comunitário de Saúde da Equipe mínima, a Política coloca em risco a prestação de uma assistência integrada à saúde da população (MOROSINI, FONSECA e LIMA, 2017; PINTO e GIOVANELLA, 2018).

Outro ponto é a definição de equipe mínima, onde se reconhece que a equipe deve ser formada apenas por médico, enfermeiro, auxiliar ou técnico de enfermagem, podendo ou não agregar outros profissionais. A nova PNAB permite flexibilização da carga horária de trabalho com dedicação parcial à Atenção Primária, utilizando-se o discurso de suprimento de demanda de profissionais em áreas com escassez profissional (FONTENELLE, 2012; BRASIL, 2017; MOROSINI, FONSECA e LIMA, 2017; CECILIO e REIS, 2018; ALMEIDA, 2018).

Esta flexibilização é criticada, pois permite ao profissional adquirir múltiplos vínculos, podendo desencadear em prestação de serviço com menor vínculo entre a população de referência e a equipe ((FONTENELLE, 2012; CECILIO e REIS, 2018). Essa flexibilização da 
carga horária, com a cobertura, evidenciam pouca preocupação com mudanças no modelo de saúde e mera redução de custos, desfavorecendo-se a longevidade da assistência (MOROSINI, FONSECA e LIMA, 2017; PINTO e GIOVANELLA, 2018).

Discute-se também a relativização da cobertura e do número de ACS - não é mais obrigatória a cobertura universal pelos ACS de todas as áreas. Apenas áreas de risco e vulneráveis deverão ser cobertas 100\% (MOROSINI, FONSECA e LIMA, 2017; CECILIO e REIS, 2018). O ACS, também, não é mais essencial na equipe mínima da $\mathrm{AB}$, fato que tem gerado dúvidas a respeito da relevância do papel do ACS e garantia de universalidade de acesso dos usuários (MOROSINI, FONSECA e LIMA, 2017).

O caráter "punitivo" das avaliações é demonstrado - a nova PNAB atribuiu remuneração adicional a depender dos resultados obtidos em avaliações, atividades de monitoramento. Este fato gerou insatisfação ao ignorar as limitações de cada equipe bem como por impossibilitar as condições legitimas de trabalho e troca de saberes (MOROSINI e FONSECA, 2017; MOROSINI, FONSECA e LIMA, 2017).

Diante do exposto observa-se que muitos são os desafios enfrentados pelo SUS e que corroboram com a sua desconstrução (MOROSINI, FONSECA e LIMA, 2017). De modo geral observa-se que as ações associadas à nova PNAB induzem ao sucateamento do SUS e subfinanciamento do mesmo, reduzindo-se a importância da Atenção Básica (MOROSINI, FONSECA e LIMA, 2017; PINTO e GIOVANELLA, 2018). Desta forma, faz-se necessário a organização de mecanismos de defesa deste pilar da assistência à saúde, através de maior participação social nos debates referentes à assistência à saúde, para que os direitos sejam garantidos de forma efetiva e universalizada (MOROSINI, FONSECA e LIMA, 2017; ALMEIDA, 2018; COSTA, 2016).

\section{Conclusão}

Apesar dos avanços inquestionáveis, em Saúde Pública, nas últimas décadas no que tange aos aspectos acessibilidade e universalização da assistência observou-se, através desta revisão de literatura que, há um consenso entre os autores, de que as mudanças promovidas pela 
nova PNAB na dinâmica dos Serviços de Saúde serão negativas gerando-se implicações significativas na dinâmica dos Serviços.

O propósito dessa nova PNAB e outras medidas adotadas, como a PEC 95/2016, evidenciam o descaso e descompromisso com a Saúde Pública. A tendência é que estas medidas corroborem com a desconstrução do SUS através da limitação de recursos e mudança de um modelo assistencial para um modelo de saúde hospitalocêntrico dicotômico ao modelo de Atenção à Saúde vigente.

Nitidamente as mudanças realizadas, nos últimos tempos, revelam um descompromisso com a Saúde Pública exigindo-se uma maior participação popular. Diante desse panorama constata-se a necessidade de maior compromisso dos membros da sociedade civil para garantia dos direitos adquiridos.

\section{Referências}

ALMEIDA PFD, SANTOS AMD. Primary Health Care: care coordinator in regionalized networks? Revista de Saúde Pública. 2016;50:80. http://dx.doi.org/10.1590/s15188787.2016050006602.

ALMEIDA PFD. Atenção primária à saúde no Brasil e os 40 anos de Alma-Ata: reconhecer os desafios para seguir adiante. Cadernos de Saúde Pública. 2018;34(8). doi: 10.1590/0102$311 X 00136118$.

BARBIANI R, DALLA NORA CR, SCHAEFER R. Práticas de enfermagem no contexto da atenção básica: uma revisão de escopo. Rev. Latino-Am. Enfermagem. 2016;24:e2721. http://dx.doi.org/10.1590/1518-8345.0880.2721.

BRASIL. Portaria $n^{\circ} 2.436$, de 21 de setembro de 2017. Diário Oficial da República Federativa do Brasil 2017; 5 set.

CARVALHO MND, GIL CRR, COSTA EMOD, SAKAI MH, LEITE SN. Necessidade e dinâmica da força de trabalho na Atenção Básica de Saúde no Brasil. Ciência \& Saúde Coletiva. 2018;23(1):295-302. http://dx.doi.org/10.1590/1413-81232018231.08702015.

CECILIO LCDO, REIS AACD. Apontamentos sobre os desafios (ainda) atuais da atenção básica à saúde. Cadernos de Saúde Pública. 2018;34(8). http://dx.doi.org/10.1590/0102$311 \times 00056917$. 
CECILIO LCDO, REIS AACD. Atenção básica como eixo estruturante do SUS: quando nossos consensos já não bastam! Cadernos de Saúde Pública. 2018;34(8). https://doi.org/10.1590/0102-311X00136718.

COSTA NDR. A Estratégia de Saúde da Família, a atenção primária e o desafio das metrópoles brasileiras. Ciência \& Saúde Coletiva. 2016;21(5):1389-98. http://dx.doi.org/10.1590/141381232015215.24842015 .

DOMINGOS CM, NUNES EFPA, CARVALHO BG, MENDONÇA FF. A legislação da atenção básica do Sistema Único de Saúde: uma análise documental. Cad Saúde Pública. 2016;32(3):1-13. http://dx.doi.org/10.1590/0102-311X00181314.

FERTONANI HP, PIRES DEPD, BIFF D, SCHERER MDDA. Modelo assistencial em saúde: conceitos e desafios para a atenção básica brasileira. Ciência \& Saúde Coletiva. 2015;20(6):1869-78. http://dx.doi.org/10.1590/1413-81232015206.13272014.

FONTENELLE LF. Mudanças recentes na Política Nacional de Atenção Básica: uma análise crítica. Rev bras med fam comunidade. 2012;7(22):5-9. https://doi.org/10.5712/rbmfc7(22)417.

MARANHÃO T, MATOS IB. Vivências no Sistema Único de Saúde (SUS) como marcadoras de acontecimento no campo da Saúde Coletiva. Interface. 2017;22(64):55-66. http://dx.doi.org/10.1590/1807-57622016.0091.

MARIANO CM. Emenda constitucional 95/2016 e o teto dos gastos públicos: Brasil de volta ao estado de exceção econômico e ao capitalismo do desastre. Revista de Investigações Constitucionais. 2017;4(1):259-81. http://dx.doi.org/10.5380/rinc.v4i1.50289

MENDES A. A saúde pública brasileira no contexto da crise do Estado ou do capitalismo? Saúde e Soc. 2015;24(suppl 1):66-81. http://dx.doi.org/10.1590/S0104-12902015S01006.

MOREIRA ÉCDR, O'DWYER G. An analysis of actions to promote health in underprivileged urban areas: a case in Brazil. BMC Family Practice. 2013;14(1). https://doi.org/10.1186/14712296-14-80.

MOROSINI MVGC, FONSECA AF. Revisão da Política Nacional de Atenção Básica numa hora dessas? Cad. Saúde Pública. 2017;33(1):1-4. http://dx.doi.org/10.1590/0102$311 \times 00206316$.

MOROSINI, Márcia Valéria Guimarães Cardoso; FONSECA, Angélica Ferreira; LIMA, Luciana Dias de. Política Nacional de Atenção Básica 2017: retrocessos e riscos para o Sistema Único de Saúde. Saúde Debate. 2018;42(16):11-24. http://dx.doi.org/10.1590/01031104201811601.

PINTO LF, GIOVANELLA L. Do Programa à Estratégia Saúde da Família: expansão do acesso e redução das internações por condições sensíveis à atenção básica (ICSAB). Ciência \& Saúde Coletiva. 2018;23(6):1903-14. http://dx.doi.org/10.1590/1413-81232018236.05592018. 
ROCHA FRF, MACÁRIO E. O impacto da EC 95/2016 e da PEC 287/2016 para a Previdência Social brasileira. Ser Social. 2017;18(39):444-60.

ROSSI P, DWECK E. Impactos do novo regime fiscal na saúde e educação. Cad. Saúde Pública. 2016;32(12):1-5. http://dx.doi.org/10.1590/0102-311x00194316.

VIEIRA FS, BENEVIDES RPS. O Direito à Saúde no Brasil em Tempos de Crise Econômica, Ajuste Fiscal e Reforma Implícita do Estado. Revista de Estudos e Pesquisas sobre as Américas. 2016;10(3):1-28. http://dx.doi.org/10.21057/repam.v10i3.21860

\section{Como citar este artigo (Formato ABNT):}

FERREIRA, Juvenildo Santos Ferreira; LADEIA, Luiz Eduardo de Góes. As Implicações da Política Nacional de Atenção Básica (PNAB) na Dinâmica dos Serviços de Saúde. Id on Line Rev.Mult. Psic., 2018, vol.12, n.42, p. 681-695. ISSN: 1981-1179.

Recebido: 15/10/2018;

Aceito: 17/10/2018 